Association for Information Systems AIS Electronic Library (AISeL)

Wirtschaftsinformatik Proceedings 2005

Wirtschaftsinformatik

February 2005

\title{
Transaktionsautomatisierung im E-Learning
}

Markus Schmees

Universität Oldenburg

Follow this and additional works at: http://aisel.aisnet.org/wi2005

\section{Recommended Citation}

Schmees, Markus, "Transaktionsautomatisierung im E-Learning" (2005). Wirtschaftsinformatik Proceedings 2005. 47.

http://aisel.aisnet.org/wi2005/47

This material is brought to you by the Wirtschaftsinformatik at AIS Electronic Library (AISeL). It has been accepted for inclusion in Wirtschaftsinformatik Proceedings 2005 by an authorized administrator of AIS Electronic Library (AISeL). For more information, please contact elibrary@aisnet.org. 
In: Ferstl, Otto K, u.a. (Hg) 2005. Wirtschaftsinformatik 2005: eEconomy, eGovernment, eSociety; 7. Internationale Tagung Wirtschaftsinformatik 2005. Heidelberg: Physica-Verlag

ISBN: 3-7908-1574-8

(C) Physica-Verlag Heidelberg 2005 


\title{
Transaktionsautomatisierung im E-Learning
}

\author{
Markus Schmees \\ Universität Oldenburg
}

Zusammenfassung: Umsetzung und Durchführung beliebiger E-LearningMaßnahmen sind i.d.R. mit hohen Kosten verbunden. Deshalb sind Erlösmodelle notwendig, die helfen, auftretende Kosten auszugleichen. Bisher fehlt ein Ansatz, derartige Erlösmodelle in elektronische Lernabläufe und insbesondere in Lernplattformen zu integrieren. In dieser Arbeit wird daher ein Instrument konzipiert, dass den Schutz von Funktionalität mit einer automatisierten Durchführung finanzieller Transaktionen kombiniert und mit der Ausführung einer Funktion verbindet. Aufgrund seiner allgemeinen Gestaltung ist dieses Instrument unabhängig von der Art eingesetzter digitaler Lernressourcen und der persönlichen Kontoführung Beteiligter. Es ermöglicht eine Berücksichtigung didaktischer, organisatorischer und ökonomischer Vorgaben und ist damit eine Testumgebung zur Erprobung verschiedenartiger Erlösmodelle im E-Learning.

Schlüsselworte: E-Learning, Finanzielle Transaktionen, Lernmanagementsysteme, Umsetzung von Erlösmodellen, Integration von Geschäftsprozessen

\section{Einleitung}

Lernen ist ein aktiver Prozess, bei dem sich Lernende neues Wissen aneignen [BaPa99]. Aufgrund eigener und zumeist wiederholter Aktivitäten erzielen sie eine meist dauerhafte Verhaltensänderung [Stei01]. Das Lernergebnis repräsentiert eine durch diesen Lernprozess verursachte Veränderung von Lernenden, die aus der Umwandlung von Informationen in verfügbares Wissen oder Können resultiert [Weng00]. Ein Lernkontext fasst Faktoren zusammen, die Auswirkungen auf das Lernergebnis haben. Das sog. E-Learning bezeichnet einen zielgerichteten Einsatz von IKT im Lernkontext [Mina02]. Darin eingesetzte Lernressourcen wie Scripte, Lerneinheiten oder tutorielle Dienste liegen i.d.R. in digitaler Form vor, sind also über Netzwerke hinweg ansprechbar und abrufbar. Dabei handelt es sich um digitale Produkte, näher charakterisiert in [Luxe01] oder [Bole02], und um digitale Dienstleistungen, die z.B. [BrSt02] näher beschreiben. Aufgrund des digitalen Charakters ist kein direkter Kontakt zwischen Anbieter und Empfänger nötig. Lernende können auf diese Weise Lernangebote unabhängig von Ort oder Zeit nutzen. Dies geschieht i.d.R. mit Hilfe von Lernmanagementsystemen (LMS), die auch als Lernportale oder -plattformen bekannt sind [Bau $\left.{ }^{+} 02\right]$. Während digitale 
Dienstleistungen auch eigenständig vorkommen, sind digitale Produkte immer mit digitalen Dienstleistungen verbunden, z.B. zur Recherche oder Auslieferung. Daher genügt zur Berücksichtigung von Lernressourcen und -aktivitäten im ELearning eine Betrachtung digitaler Dienstleistungen. LMS bündeln diese und stellen sie ihren Nutzern in Form von Funktionen zum Ansprechen zur Verfügung.

Weiterhin interagieren im E-Learning verschiedene Beteiligte, die Dienstleistungen sowohl erbringen (z.B. Lehrende, Tutoren), als auch beanspruchen (z.B. Lernende). Ist das Angebot kostenlos, sind Beteiligte eher bereit, fremde Dienstleistungen zu nutzen als eigene anzubieten. Insbesondere unter gleichberechtigten Beteiligten ist eine Ausnutzung fremder Ressourcen, das sog. Free-Riding, die Folge [AdHu00]. Ein kostenpflichtiges Angebot beugt diesem Missbrauch vor, da ein Anbieter bei übermäßiger Beanspruchung neue Ressourcen erwerben kann [Gol $\left.{ }^{+} 01\right]$. Voraussetzung für den Handel sind Geschäftsmodelle, die Geschäftsund Ertragslogik eines Unternehmens repräsentieren und damit die Verbindung zwischen Geschäftsstrategie und -prozessen darstellen [Oste04]. Erlösmodelle sind Teil eines Geschäftsmodells und bilden darin vorgesehene Erlösflüsse $a b$, z.B. in Form finanzieller Transaktionen. Diese übermitteln in ihrer einfachen Form einen bestimmten Betrag vom Konto eines Senders zum Konto eines Empfängers. In dieser Arbeit wird davon ausgegangen, dass keine Transaktionskosten Markt, Handel oder Verhalten der Beteiligten beeinflussen. Grundlage für einen Handel im E-Learning ist eine funktionierende kommerzielle Infrastruktur, die den Schutz digitaler Güter sicherstellt und eine Umsetzung entsprechender Erlösmodelle erlaubt.

Ziel dieser Arbeit ist nicht die Entwicklung eines neuen Geschäftsmodells für das E-Learning, sondern die Konzeption einer konfigurierbaren Testumgebung, die Umsetzung und Erprobung verschiedenartiger Erlösmodelle auf Basis bereits eingesetzter LMS erlaubt. Zur Veranschaulichung sei folgendes Szenario gegeben. Angenommen, eine Hochschule setzt zur Unterstützung der Präsenzlehre ein LMS ein. Mit Hilfe eines Forums soll eine intensive tutorielle Betreuung der Lernenden stattfinden. Um Kosten für Tutoren zu reduzieren und gleichzeitig ihre Motivation zu belohnen, verlangt ein Dozent zur Beantwortung von Fragen im Forum eine Gebühr. Davon geht eine Hälfte an den Dozenten, die andere Hälfte bekommt der Tutor, der als erster eine Frage beantwortet. Das Einfügen von Nachrichten in ein Forum ist eine Funktion, die ein LMS bietet. Dennoch ist die Verknüpfung von Transaktionen mit Funktionen eines LMS sowie ihre bedingte und automatisierte Durchführung im elektronischen Lernkontext bisher nicht vorgesehen.

Diese Arbeit stellt in Abschnitt 2 zunächst Gründe und Ziele einer Integration von Geschäftsprozessen in das E-Learning vor. Abschnitt 3 beschreibt ein Konzept, das die Modellierung und Steuerung von Transaktionen sowie ihre automatisierte Abwicklung erlaubt. Darauf aufbauend stellt Abschnitt 4 am Beispiel des LMS Stud.IP die Vorgehensweise bei der Integration des Konzeptes in ein E-LearningSystem vor. Verwandte Ansätze und Arbeiten betrachtet Abschnitt 5, bevor Abschnitt 6 mit einer Zusammenfassung und einem Ausblick schließt. 


\section{Motivation und Ziele}

Der Schutz digitaler Dienstleistungen und die Steuerung einfacher finanzieller Transaktionen sind Voraussetzung für die Umsetzung und Erprobung von Erlösmodellen, Integration geschäftlicher Prozesse in den Lernablauf, Realisierung komplexer Transaktionen, Sicherung von Qualität sowie wirtschaftliches Handeln im E-Learning. Die einzelnen Begründungen werden nachfolgend skizziert.

- Umsetzung von Erlösmodellen: Bei Einführung und Anwendung von ELearning entstehen Kosten, die i. A. sowohl schwer zu bestimmen als auch voneinander abzugrenzen sind [Moon97]. Eine Unterscheidung von einmaligen, laufenden, fixen und variablen Kosten sowie Kosten für Entwicklung, Durchführung, Infrastruktur und Overhead findet sich in [Leh $\left.{ }^{+} 03\right]$. Typ und Höhe anfallender Kosten hängen i. d. R. von Art, Ausprägung und Umfang einer E-Learning-Maßnahme ab, konkrete Kalkulationen stellen z.B. [Rumb01] oder [Jung03] auf. Voraussetzung zur kontinuierlichen Bereitstellung von E-Learning-Diensten sind Erlösmodelle, die eine nachhaltige Wertschöpfung ermöglichen [Timm98]. Abgesehen von übergeordneten Ansätzen (wie z.B. Subventionierung) fehlt bisher ein Instrument, das eine einfache Umsetzung und Erprobung dieser Erlösmodelle im elektronischen Lernkontext erlaubt.

- Integration von Geschäftsprozessen: E-Commerce basiert ebenso wie ELearning auf dem gezielten Einsatz von IKT, Geschäftsmodelle bringen beide Konzepte zusammen [HaOt02]. Aus ökonomischer Sicht ist darauf aufbauend die Integration geschäftlicher Prozesse in elektronische Lernabläufe möglich. Eine Lernplattform kann dann, z.B. abhängig vom Verhalten der Nutzer oder auf Basis von Ereignissen, finanzielle Transaktionen vornehmen. Voraussetzung ist ein Instrument, das Modellierung und automatisierte Durchführung von Transaktionen innerhalb von Lernplattformen erlaubt.

- Modellierung komplexer Transaktionen: Das Erbringen einer einfachen Dienstleistung erfolgt ohne Beteiligung weiterer Dienstleistungen. Komplexe Dienstleistungen bestehen aus einer Menge einfacher oder komplexer Dienstleistungen. Eine kostenpflichtige komplexe Dienstleistung kann somit weitere kostenpflichtige Dienstleistungen beinhalten. Im E-Learning-Bereich erfolgt das Ansprechen einer Dienstleistung durch Aufruf einer zugehörigen Funktion. Ein Instrument ist darum notwendig, das neben der Ausstattung einer Funktion mit einer Transaktionslogik auch die Durchführung komplexer Transaktionen sicherstellt.

- Sicherung von Qualität: Der Einsatz von IKT trägt zwar zur Sicherung der Qualität im E-Learning bei [ScHe02], dennoch sehen einer Umfrage zufolge $61 \%$ der Befragten die Qualität von E-Learning-Angeboten als ausreichend bis schlecht an [Mass02]. Eine transparente Darstellung von Preis und Leistung ermöglicht eine Form der Qualitätssicherung. So ist im Rahmen von 
Verbraucherschutz und Markttransparenz davon auszugehen, dass die Sicherung von Qualität durch Marktmechanismen wie z.B. Marktbereinigung erfolgt [Bal $\left.{ }^{+} 02\right]$. Dazu notwendig ist ein Instrument, das neben der Modellierung und Durchführung von Transaktionen auch die Darstellung von Voraussetzungen übernimmt und ihre Erkundung durch Dritte erlaubt.

- Wirtschaftliches Handeln: Im kostenpflichtigen elektronischen Lernkontext können persönliche Mittel, z.B. in Form von Spielgeld oder Punkten, eingeschränkt sein. Um dennoch größtmöglichen Lernerfolg zu erzielen, sind Beteiligte gezwungen, eigene Mittel zielgerichtet einzusetzen. Neben der Möglichkeit wirtschaftlich zu handeln erhalten sie damit eine größere Eigenverantwortung für den persönlichen Lernerfolg. Gehen Lernende verschwenderisch mit ihren Mitteln um und nehmen z.B. Dienste wahr, die nicht zum Lernerfolg führen, machen sie dies auf eigene Rechnung. In diesem Fall müssen sie z.B. an anderer Stelle Einsparungen vornehmen, um dennoch ein benötigtes Lernergebnis zu erzielen. Dies reduziert einerseits die Ausnutzung von Ressourcen, die nicht zwingend zum Lernerfolg beitragen. Andererseits erhalten Nutzer die Möglichkeit, durch selbstbestimmte Verteilung von Mitteln Lernressourcen zu nutzen, die ihre persönliche Interessenlage repräsentieren. Voraussetzung ist ein Instrument, das ein Ansprechen von Lernressourcen, die Steuerung von Transaktionen und die persönliche Kontoführung beteiligter Nutzer zusammenbringt.

Um diese Ziele zu erreichen ist ein Instrument notwendig, das die von einem LMS angebotenen Funktionen (und damit Lernressourcen) vor unbefugten Zugriffen schützt und die Modellierung, Steuerung sowie automatisierte Durchführung komplexer Transaktionen im elektronischen Lernkontext erlaubt. Der folgende Abschnitt stellt das zugehörige Konzept vor.

\section{Konzeption}

Voraussetzung zur Verknüpfung finanzieller Transaktionen mit der Erbringung digitaler Dienstleistungen im E-Learning-Bereich ist ein System, das diese Dienstleistungen in Form von Funktionen zum Ansprechen anbietet. Dabei handelt es sich i.d.R. um ein LMS. Das in dieser Arbeit vorgestellte Konzept beruht auf einer solchen Menge von Funktionen (und dadurch repräsentierte Lernaktivitäten) und erfüllt darauf aufbauend insbesondere zwei Anforderungen. Zum einen schützt es den Zugriff auf diese Funktionen und erlaubt ihre Ausführung nur berechtigten Nutzern oder Systemen. Zum anderen verknüpft es die Ausführung einer Funktion mit der Durchführung finanzieller Transaktionen.

Schutzmechanismen sorgen dafür, dass nur befugte Nutzer oder Systeme eine Funktion ausführen dürfen. Eine Transaktionslogik hingegen bestimmt Sender, 
Empfänger und Höhe einer finanziellen Transaktion und übernimmt ihre Durchführung. Die Integration von Schutzmechanismen und Transaktionslogik in eine Menge bestehender Funktionen muss jedoch bestimmte Anforderungen erfüllen. So darf ihr Zufügen die ursprüngliche Funktionalität einer Funktion nicht verändern und muss ihre Implementierung weitgehend erhalten. Der Aufruf einer Funktion darf nicht verändert werden, um ein problemloses Ansprechen zu gewährleisten und eine Anpassung zugreifender Anwendungen zu vermeiden. Um problemlos auf Änderungen geschäftlicher Vorgaben im Lernkontext zu reagieren, müssen Schutz und Freigabe beliebiger Funktionen auch im Nachhinein ohne großen Aufwand möglich sein. Ein Zielsystem muss dazu sowohl ursprüngliche als auch erweiterte Funktionen verarbeiten können. Um Funktionen vor unbefugten Zugriffen zu schützen, muss eine Ausstattung mit Schutzmechanismen unabhängig von der Ausstattung mit Transaktionslogik möglich sein. Um jedoch ein unbeabsichtigtes Ansprechen und Auslösen von Transaktionen zu vermeiden und das dazu notwendige Einverständnis eines Nutzers sicherzustellen, müssen die mit Transaktionslogik ausgestatteten Funktionen gleichzeitig Schutzmechanismen erhalten. Zudem ist die Verknüpfung mehrerer Transaktionen mit der Ausführung einer Funktion vorzusehen, um größtmögliche Flexibilität bei der Modellierung von Transaktionen zu erhalten. Um Sender, Empfänger und Höhe einer Transaktion auf bestimmte Bedingungen abzubilden, die sich z.B. während der Ausführung einer Funktion ergeben, müssen Modellierung und Durchführung bedingter Transaktionen möglich sein. Zudem darf eine Durchführung erst nach erfolgreicher Ausführung einer Funktion erfolgen, um im Fall eines Fehlers die Transaktion zu vermeiden.

Abschnitt 3.1 beschreibt die grundlegende Idee, um die genannten Anforderungen zu erfüllen, nämlich eine Ausstattung von Funktionen mit Prolog und Epilog. Auf diese Weise ist es möglich, bestehende Funktionalität mit einer Rechteverwaltung und interner Abrechnung zu verbinden. Die Modellierung finanzieller Transaktionen beschreibt Abschnitt 3.2. Ihre Kombination mit Rechten und Gültigkeiten erfolgt über Lizenzen, näher aufgeführt in Abschnitt 3.3. Abschnitt 3.4 beschreibt darauf aufbauend die Vorgehensweise bei der Zuteilung und Beanspruchung von Lizenzen. Abschnitt 3.5 schließlich zeigt eine Übersicht über die angedachte Architektur des Gesamtsystems.

\subsection{Ausstattung von Funktionen mit Prolog und Epilog}

Aus den vorab dargestellten Anforderungen geht hervor, dass Schutzmechanismen bereits vor Ausführung einer Funktion greifen müssen, die Transaktionslogik jedoch erst nach ihrer erfolgreichen Ausführung zum Einsatz kommt. Die grundlegende Idee des vorgestellten Konzeptes ist daher, ursprüngliche Funktionen mit einem Prolog auszustatten, der ihren Schutz übernimmt, sowie einem Epilog, der für die finanziellen Transaktionen zuständig ist. 
Der Unterschied zwischen ursprünglichen und erweiterten Funktionen ist auf der linken Seite in Abb. 1 illustriert. Ursprüngliche Funktionen repräsentieren die von einem bestehenden System angebotene Funktionalität. Zugriff darauf ist im Rahmen des Systems möglich, ein zusätzlicher Schutz bzw. eine Verbindung dieser Funktionen mit der Durchführung von Transaktionen i.d.R. nicht vorgesehen. Erweiterte Funktionen sind ursprüngliche Funktionen, die zusätzlich mit Prolog und Epilog ausgestattet sind. Auf diese Weise bleibt ihre Funktionalität erhalten, ebenso die Art ihres Aufrufs. Während ein Prolog in erster Linie für die Klärung von Voraussetzungen zuständig ist, muss der Epilog nach Ausführung der Funktion bestimmte Nachbedingungen herstellen.
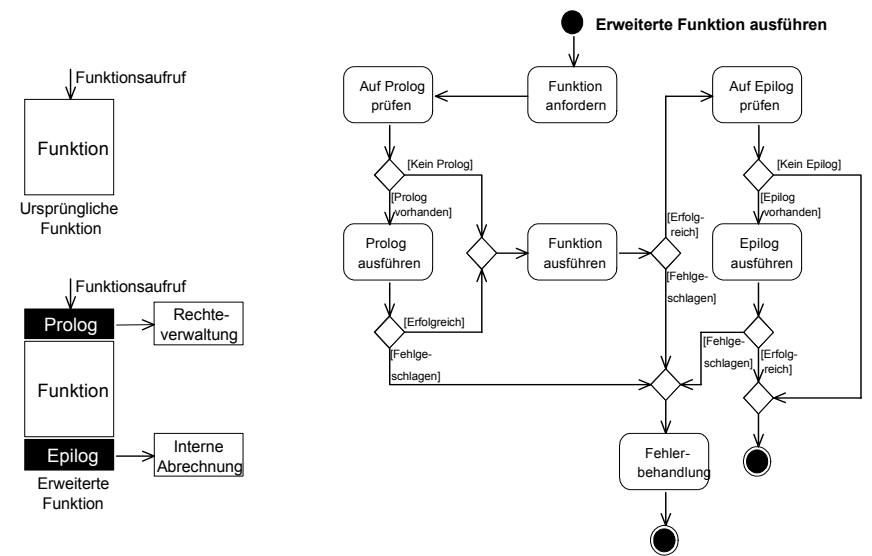

Abbildung 1: Ausstattung einer Funktion mit Prolog und Epilog

Die Vorgehensweise bei der Ausführung einer erweiterten Funktion ist in Form eines UML-Aktivitätsdiagramms auf der rechten Seite in Abb. 1 dargestellt. Nach Anforderung einer Funktion ist zunächst festzustellen, ob ein zugehöriger Prolog existiert. In diesem Fall klärt er notwendige Vorbedingungen ab, z.B. ob bestimmte Nutzer oder Systeme das Recht haben, die aufgerufene Funktion auszuführen. Voraussetzung dazu ist eine Rechteverwaltung, die eine Verbindung zwischen Rechten, Funktionen und Nutzern herstellt. Weiterhin prüft der Prolog, ob ein Nutzer seine Zustimmung zur Transaktion gegeben hat. Er versucht die Attribute spezifizierter Transaktionen vorab zu bestimmen und prüft, ob ihre Durchführung im Nachhinein überhaupt möglich ist. Sind sämtliche Vorbedingungen erfüllt und das Recht auf Zugriff vorhanden, folgt die Ausführung der Funktion. Ansonsten bricht der Prolog mit einem Fehler ab. Treten hingegen innerhalb der Funktion Fehler auf, bricht sie ihre Ausführung ab und der anschließende Epilog wird nicht erreicht. Somit findet auch keine zugehörige Transaktion statt. Ansonsten folgt im Anschluss an die Ausführung die Ermittlung des zugehörigen Epilogs und, wenn dieser existiert, seine Ausführung. Dort erfolgt das endgültige Festlegen von Transaktionsattributen und die Durchführung zugehöriger Transaktionen. Voraus- 
setzung dazu ist eine interne Abrechnung, die interne Konten der beteiligten Nutzer oder Systeme verwaltet.

Der Prolog dient also dem Schutz von Funktionalität, der Epilog ist für die automatisierte Durchführung der Transaktionen zuständig. Da ein bezahlender Nutzer immer sein Einverständnis zur Durchführung einer Transaktion geben muss, sind Funktionen, die nur einen Epilog aber keinen Prolog besitzen, im Rahmen des hier vorgestellten Konzeptes nicht vorgesehen. Ebenso wurde von der dynamischen Änderung der Transaktionsattribute im Verlauf der Funktionsausführung abgesehen, da ein zahlender Nutzer am Ende der Funktion dieser veränderten Transaktion erneut zustimmen müsste. Eine Möglichkeit zur Spezifikation und Darstellung bedingter finanzieller Transaktionen beschreibt der folgende Abschnitt.

\subsection{Modellierung bedingter finanzieller Transaktionen}

Grundlage dieser Arbeit ist die automatisierte Durchführung finanzieller Transaktionen, und zwar nach Ausführung einer auslösenden Funktion. Der Epilog stellt dabei einen Zusammenhang zwischen der Funktion und einer Menge zugehöriger Transaktionen her. Auf diese Weise kann die Spezifikation finanzieller Transaktionen unabhängig von bestimmten Funktionen erfolgen. Die Durchführung einer Transaktion ist nicht in jedem Fall notwendig, sondern hängt von Vorbedingungen ab, die sich im Verlauf der Funktionsausführung ergeben können. Eine Spezifikation dieser Vorbedingungen ist damit ebenfalls Teil einer Transaktion. Um Funktionen und Transaktionen einander zuzuordnen, muss die Spezifikation von Transaktionen und Vorbedingungen vorab erfolgen.

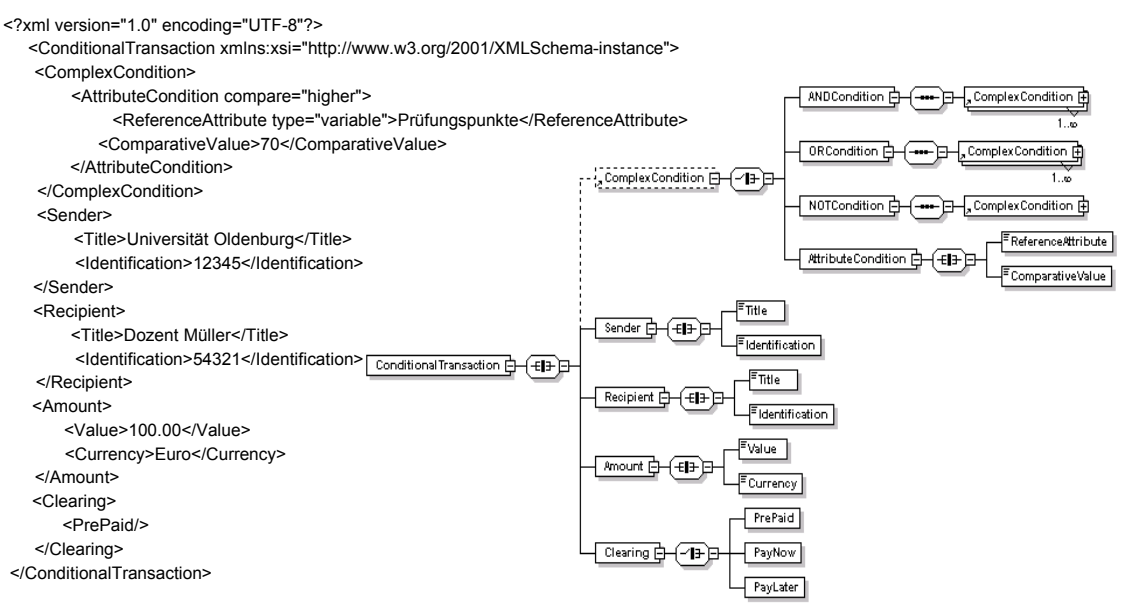

Abbildung 2: Spezifikation von Transaktionen 
Zur Spezifikation bedingter finanzieller Transaktionen wurde ein XML-Schema geschaffen, das Abb. 2 auf der rechten Seite in XMLSpy ${ }^{1}$-Notation darstellt. Durch das XML-Schema existiert eine einheitliche Darstellung bedingter finanzieller Transaktionen. Auf diese Weise ist einerseits die Spezifikation von Transaktionen durch dritte Stellen, z.B. eine Hochschule, möglich. Andererseits erlaubt diese Form der Darstellung auch eine einfache Veröffentlichung von Transaktionen sowie ihre Erkundung durch Nutzer oder Organisationen, die an ihrer Durchführung beteiligt sind. Die Elemente einer bedingten finanziellen Transaktion, dargestellt als ConditionalTransaction, sind die Folgenden:

- ComplexCondition: Spezifiziert die Vorbedingung, die erfüllt sein muss, damit die zugehörige Transaktion durchgeführt wird. Diese Angabe ist optional - wird sie weggelassen, erfolgt die Durchführung der Transaktion immer. Eine komplexe Vorbedingung kann aus weiteren komplexen oder einfachen Bedingungen bestehen, die über logische UND, ODER oder NOT-Operatoren miteinander verknüpft sind. Eine einfache Bedingung entspricht der bereits im Umfeld von Mehrbenutzerkontexten von [Habe03] als wichtig identifizierten Attributbedingung. Diese erlaubt den Vergleich eines Referenzattributs (ReferenceAttribute) aus dem Kontext des ursprünglichen Systems mit einem Vergleichswert (ComparativeValue). Voraussetzung dazu ist die Verwendung einer Attributauswertung, die Abfrage und Vergleich von Attributen oder Zuständen des zugrunde liegenden Systems ermöglicht.

- Sender/Recipient: Erlaubt Angaben über Sender und Empfänger einer finanziellen Transaktion, insbesondere den Namen oder eine Beschreibung (Title) sowie einen Identifikator (Identification), um eine eindeutige Zuordnung zu einem internen Konto vornehmen zu können. Auch Gruppen, Kurse, Systeme oder weitere Organisationen können ein Konto besitzen und so in den Abrechnungsprozess integriert werden. Voraussetzung dazu ist die Verwaltung interner Konten. Diese ist im vorgestellten Fall durch die interne Abrechnung realisiert.

- Amount: Gibt die Höhe (Amount) der zugehörigen Transaktion an, ebenso die Währung (Currency), in der diese Transaktion stattfinden soll.

- Clearing: Bestimmt Art und Weise, in der ein Ausgleich des internen Kontos eines Senders stattfinden soll. Erfolgt die Angabe PrePaid, muss dieses Konto den zu zahlenden Betrag bereits enthalten, damit die Transaktion stattfinden kann. Bei PayNow erfolgt ein Ausgleich des internen Kontos mit dem persönlichen Konto eines Nutzers zum Zeitpunkt der Transaktion. Ist hingegen PayLater angegeben, erfolgt dieser Ausgleich später, z.B. am Ende eines Monats. Das Konto darf in diesem Fall auch mit negativen Beträgen belastet sein. Voraussetzung ist eine externe Bezahlung, die in Verbindung mit der internen Abrechnung den Ausgleich interner Konten erlaubt.

$1 \quad$ http://www.xmlspy.com/ 
Auf der linken Seite in Abb. 2 ist ein Beispiel für eine einfache bedingte Transaktion dargestellt. In diesem Fall übermittelt die Universität Oldenburg mit der Kontonummer 12345 dem Dozenten Müller mit dem Konto 54321 einen Betrag von $€$ 100,00. Das Konto der Universität muss diesen Betrag vorab enthalten und die Transaktion wird nur ausgeführt, wenn in einer Prüfung mehr als 70 Punkte erreicht wurden, ausgedrückt durch das Attribut Prüfungspunkte.

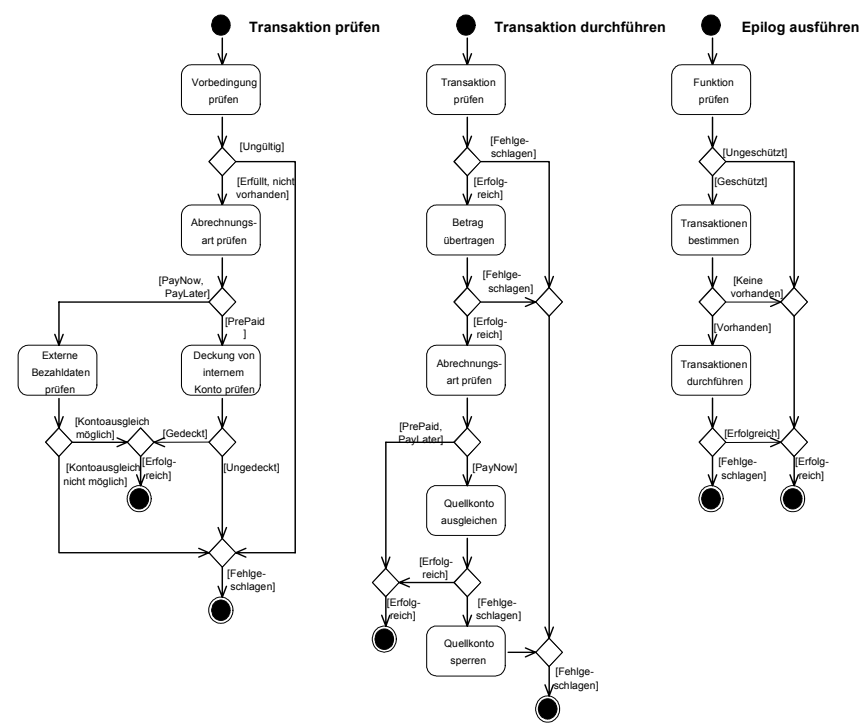

Abbildung 3: Durchführung von Transaktionen

Die Vorgehensweise bei der Ausführung eines Epilogs ist auf der rechten Seite in Abb. 3 dargestellt. Zunächst prüft der Epilog, ob die gerade ausgeführte Funktion eine geschützte Funktion ist. In diesem Fall können zugehörige Transaktionen existieren, die zunächst zu bestimmen und dann durchzuführen sind. Die Durchführung einer Transaktion ist in der Mitte auf Abb. 3 illustriert. Ihr geht eine Prüfung der Transaktion voran, dargestellt auf der linken Seite. Sind angegebene Vorbedingungen erfüllt, richtet sich die weitere Vorgehensweise nach der spezifizierten Art der Abrechnung. Im Fall von PrePaid muss der zu transferierende Betrag bereits auf dem internen Konto des zahlenden Nutzers sein. Bei PayNow oder PayLater erfolgt der Ausgleich dieses Kontos bei oder nach Durchführung der Transaktion. Auf diese Weise können z.B. geringfügige Zahlungen über einen Zeitraum gesammelt und erst dann eingefordert werden. Voraussetzung zum Ausgleich eines internen Kontos ist die Angabe gültiger Daten, die das Ansprechen eines externen Bezahlsystems ermöglichen. Die Prüfung einer Transaktion ist dann erfolgreich, wenn diese Daten gültig sind und mit ihrer Hilfe ein Ausgleich des internen Kontos möglich ist. Nach erfolgreicher Prüfung einer Transaktion überträgt der Epilog den spezifizierten Betrag vom internen Konto des Senders auf das interne Konto des Empfängers. Abhängig von der Abrechnungsart ist der Ausgleich des 
Empfängerkontos bereits erfolgt (PrePaid), findet später statt (PayLater) oder ist aktuell durchzuführen (PayNow). Schlägt dieser Ausgleich jedoch fehl, da z.B. ein externes Konto nicht hinreichend gedeckt ist, erfolgt eine Sperrung des internen Kontos, und zwar solange, bis sein Inhaber gültige Bezahldaten angibt oder diesen Ausgleich manuell vornimmt.

Mit Hilfe des vorgegebenen Formats ist es möglich, bedingte finanzielle Transaktionen zu spezifizieren. Dritte Stellen, z.B. die Nutzer einer Funktion, können auf diese Weise zugeordnete Transaktionen verstehen und resultierende Konsequenzen abschätzen. Bereits vor Ausführung einer Funktion erlauben die zugehörigen Transaktionen eine Einschätzung, ob ihre Durchführung überhaupt möglich ist. Ist dies nicht der Fall, erhält der Nutzer keinen Zugriff auf die Funktion. Für die Spezifikation ist eine Komponente vorgesehen, ebenso für die Ausführung des Epilogs. Eine Attributkomponente übernimmt die Abfrage systeminterner Attribute und erlaubt damit die Überprüfung von Attributbedingungen. Zum Ausgleich eines internen Kontos muss das Ansprechen externer Bezahlsysteme möglich sein. Diesen Zweck erfüllt eine Bezahlkomponente. Da ein zahlender Nutzer vorab mit der Durchführung einer Transaktion einverstanden sein muss, darf die Ausführung einer Funktion nur erfolgen, wenn diese Genehmigung vorliegt. Um das Recht auf Ausführung mit aufrufenden Nutzern und Transaktionen zu verknüpfen, erfolgt ein Einsatz von Lizenzen. Der folgende Abschnitt geht näher darauf ein.

\subsection{Kapselung von Rechten und Gültigkeiten in Lizenzen}

Eine Beschränkung des Zugriffs auf Funktionen und damit auf digitale Dienstleistungen kann didaktischen, organisatorischen oder finanziellen Hintergrund haben. Während didaktische Vorgaben z.B. festlegen, welche Lerneinheiten durchgearbeitet sein müssen, bevor eine zugehörige Prüfung durchgeführt werden darf, bilden organisatorische Vorgaben z.B. generelle Ordnungen oder Regelungen einer Hochschule nach. Finanzielle Vorgaben hingegen besagen, dass z.B. Nutzer erst dann Zugriff auf bestimmte Funktionen bekommen, wenn sie zuvor dafür bezahlt oder sich mit einer anschließenden Bezahlung einverstanden erklärt haben. Hinzu kommt, dass für verschiedene Nutzer unterschiedliche Vorgaben gelten können. Nutzer oder Systeme dürfen also erst dann eine Funktion ausführen, wenn sie das Recht dazu haben und festgelegte Vorbedingungen erfüllt sind. Um abstrakte Rechte mit Personen und Gültigkeiten zu verbinden, erfolgt ein Einsatz von Lizenzen. Diese geben an, ob und wann welcher Nutzer auf welche Dienste zugreifen darf. Sie verknüpfen dieses Recht mit Gültigkeiten wie Vorbedingungen, Zielgruppen oder Verbrauchswerten. Dieser Ansatz wurde bereits bei der Konzeption kostenpflichtiger Web Services in Form verschiedener Lizenztypen erprobt [BoSc03]. Lizenzen, deren Einsatz Grundlage für eine Automatisierung von Transaktionen ist, spezifizieren im Rahmen dieser Arbeit folgende Elemente: 
- Inhaber: Besitzer verfügen über eine Lizenz und können sie, falls nötig, widerrufen oder ändern. I.d.R. sind dies Nutzer oder Organisationen, die für Kosten aufkommen, welche im Rahmen zugehöriger Transaktionen entstehen.

- Zielgruppe: Gibt konkret an, wer eine Lizenz beanspruchen und nutzen darf, z.B. einzelne Nutzer, Gruppen, Kurse oder ganze Systeme. Weiterhin sind Kombinationen daraus oder Lizenzen mit unbeschränkter Gültigkeit möglich.

- Zielfunktion: Spezifiziert die konkrete Funktion, für die eine Lizenz gilt, und zwar unter Berücksichtigung verschiedener Parameter, die zur Steuerung und zum Ansprechen der Funktion beitragen.

- Vorbedingungen: Angabe einer komplexen Vorbedingung als Grundlage zur Beanspruchung einer Lizenz. Nur wenn diese Vorbedingung erfüllt ist, ist eine Nutzung der Lizenz möglich. In Verbindung mit Zielfunktion und Nutzer realisiert eine Vorbedingung den für den aktuellen Fall spezifizierten Prolog.

- Verbrauch: Legt die Intensität fest, in der eine Lizenz beansprucht und damit eine Funktion ausgeführt werden darf. Verbrauchswerte sind z.B. Menge (PayPerUse), Zeit (PayPerTime), Parametergröße, -anzahl oder ein Ressourcenwert. Eine Kombination dieser Verbrauchsgrößen innerhalb einer Lizenz ist ebenso möglich wie unbeschränkte Lizenzen ohne Verbrauch.

- Transaktionen: Eine Menge bedingter finanzieller Transaktionen, die im Zusammenhang mit der Zielfunktion auszuführen sind. Ihre Spezifikation erfolgt im Voraus, so dass sie bei Aufruf einer Zielfunktion bereits feststehen. Ihre bedingte Ausführung entspricht dem Epilog der Zielfunktion.

Lizenzen verbinden das Recht auf Ausführung einer Funktion mit bestimmten Nutzern. Ebenso spezifizieren sie den zu Funktion und Nutzer zugehörigen Prolog und Epilog. Auf diese Weise erhält eine Funktion individuell angepasste Pro- und Epiloge, jeweils abhängig vom aufrufenden Nutzer. Eine Spezifikation von Vorbedingungen und Transaktionen kann durch dritte und unabhängige Stellen erfolgen, z.B. Fachdidakten im E-Learning-Umfeld. Da die Ausführung einer Funktion Auswirkung auf Attributwerte und damit die Vorbedingung einer Transaktion haben kann, trägt sie zu deren Steuerung bei. Lizenzen repräsentieren weiterhin das Einverständnis ihrer Inhaber zur Durchführung zugehöriger Transaktionen. Ist eine Funktion geschützt, kann sie nur der Nutzer ausführen, für den eine entsprechende Lizenz vorliegt. Der folgende Abschnitt beschäftigt sich näher mit dem Vorgang der Zuteilung und Verwendung von Lizenzen.

\subsection{Zuteilung und Verwendung von Lizenzen}

Ist eine Funktion geschützt, können Nutzer oder Systeme nur dann darauf zugreifen, wenn sie der Zielgruppe einer zugehörigen und gültigen Lizenz angehören. Bei der Zuteilung einer Lizenz erfolgt ihre Aktivierung sowie eine Zuordnung zu 
Inhabern. Eine Zuteilungskomponente nimmt diese Zuteilung automatisiert vor. Voraussetzung dazu sind Angebote, die sämtliche zur Zuteilung notwendigen Informationen darstellen. Sie enthalten insbesondere Zielfunktion, Zielgruppe, Transaktionen und Vorbedingungen. Eine Spezifikation von Angeboten beinhaltet die Auswahl angemessener Transaktionen und erfolgt durch Nutzer oder Systeme, die Verfügungsgewalt über die zugrunde liegende Zielfunktion besitzen. Im einfachen Fall sind diese Anbieter selbst Empfänger der spezifizierten Transaktionen. Akzeptiert ein Nutzer ein derartiges Angebot, erstellt die Zuteilungskomponente auf dessen Basis eine Lizenz.

Im Rahmen einer Ökonomisierung des E-Learning muss insbesondere das kostenpflichtige Angebot digitaler Lernressourcen bedacht werden. So ist z.B. nicht jeder Lehrende oder jede Hochschule bereit, Dienste oder Materialien umsonst zur Verfügung zu stellen. Eine kostenpflichtige Gestaltung von Lizenzen erlaubt dies auf einer einfachen Ebene. Die Zuteilung von Lizenzen erfolgt im kostenpflichtigen Fall nicht direkt nach Anforderung und Zustimmung durch einen Nutzer, sondern indirekt nach dessen Bewerbung über Marktmechanismen auf Basis kostenpflichtiger Angebote. Bei der direkten Erlöserzielung stimmen Zielgruppe und Inhaber einer Lizenz überein, im indirekten Fall kommen Inhaber für Transaktionen auf, die eine fremde Zielgruppe auslöst. Auf diese Weise ist eine Umsetzung einfacher Erlösmodelle im E-Learning-Bereich bereits möglich, z.B. das kostenpflichtige Herunterladen digitaler Lernmaterialien oder das Versteigern von Plätzen zu zulassungsbeschränkten Veranstaltungen.

Die Vorgehensweise bei der Zuteilung von Lizenzen ist auf der linken Seite in Abb. 4 dargestellt. Nachdem ein Nutzer entsprechende Angebote erkundet und sich für eines entschieden hat, stimmt er damit indirekt den darin enthaltenen Bedingungen und Transaktionen zu. Ist das Angebot kostenpflichtig, findet zunächst eine Preisfindung statt, z.B. als Festpreis, Verhandlung oder Auktion. Erhält der Nutzer daraufhin den Zuschlag, erfolgt die Bezahlung in Form einer einfachen Transaktion. Ist diese ebenfalls erfolgreich, erstellt das System eine Lizenz. Eine Zielgruppe erhält damit das Recht auf Ausführung der Zielfunktion. Umfasst ein Angebot mehrere Transaktionen, müssen sämtliche zahlenden Nutzer zustimmen, damit es zur Zuteilung einer Lizenz kommt. Diese werden dann als Inhaber der Lizenz aufgeführt und können sie bei Bedarf widerrufen.

Die Beanspruchung einer Lizenz erfolgt im Rahmen der Durchführung eines Prologs und ist auf der rechten Seite in Abb. 4 illustriert. Nach Aufruf einer Funktion durch einen Nutzer oder ein System folgt die Überprüfung, ob die Funktion öffentlich oder geschützt ist. Während die Freigabe einer öffentlichen Funktion direkt erfolgt, benötigt ein Nutzer zur Ausführung einer geschützten Funktion immer eine zugehörige Lizenz. Der Prolog prüft bei der Lizenz, ob sie sich auf die aufgerufene Funktion bezieht, der aufrufende Nutzer zur Zielgruppe gehört, sämtliche angegebenen Vorbedingungen erfüllt sind, der Verbrauch ausreicht und die angegebenen Transaktionen durchführbar sind. Enthält der Verbrauch einer Lizenz einen Ressourcenwert, ist erst die Ausführung der zugehörigen Funktion nö- 
tig, um diesen zu bestimmen und abzuziehen. Eine entsprechende Vorgehensweise ist in [BoSc03] dargestellt und wird im Rahmen dieser Arbeit nicht näher betrachtet. Ist eine Lizenz gültig, wird sie beansprucht, indem der ermittelte vom vorhandenen Verbrauch abgezogen wird. Hat das ebenfalls geklappt, erfolgt die Freigabe der Funktion und der Prolog ist beendet.
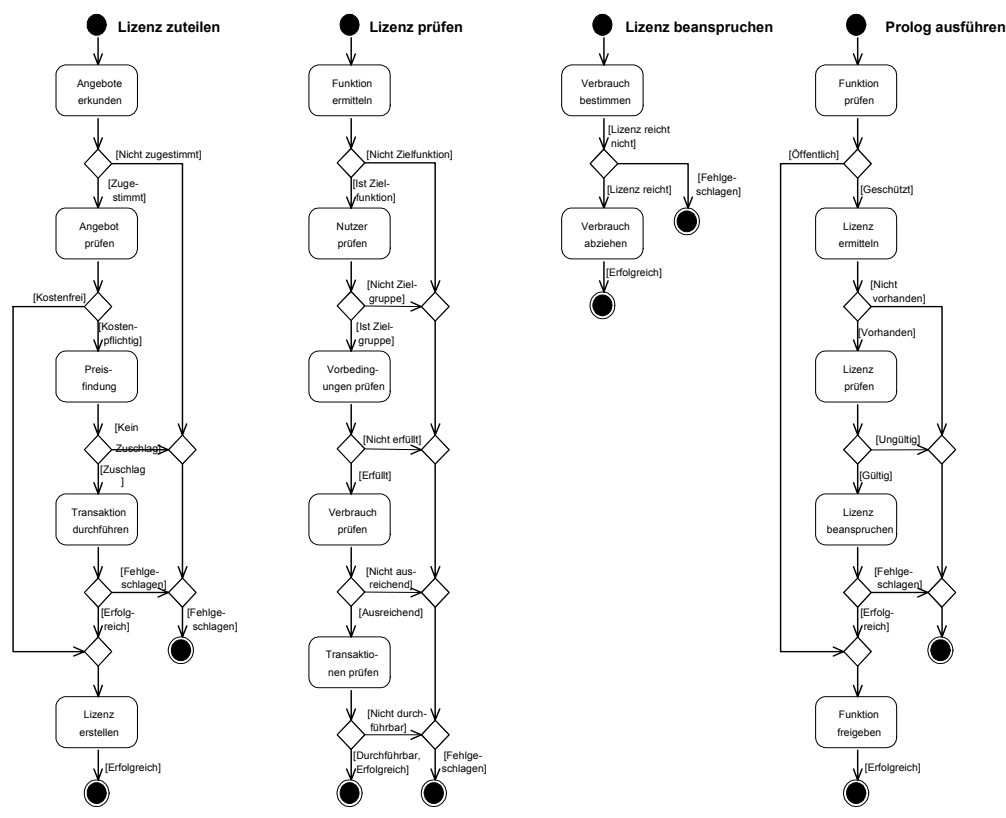

Abbildung 4: Zuteilung und Beanspruchung einer Lizenz

Eine Umsetzung komplexer Erlösmodelle, z.B. eine erfolgsabhängige Vergütung für Dozenten oder die Rückerstattung von Studiengebühren auf Basis des Lernerfolgs, ist mit Hilfe automatisierter finanzieller Transaktionen möglich. Ihre Durchführung ist im Zusammenhang mit der Ausführung einer Funktion vorgesehen, nämlich im Epilog. Dieser hat damit direkten Einfluss auf zahlende Nutzer oder Organisationen, da darin modellierte Transaktionen deren Kontostand verändern. Voraussetzung zur Durchführung einer Transaktion ist das Einverständnis der zahlenden Nutzer, die vorab zu einer Funktion zugehörige Transaktionen und deren Auswirkungen kennen müssen. Spezifikation und Veröffentlichung von Transaktionen erfolgen daher innerhalb von Angeboten. Angebote stellen damit einen $\mathrm{Zu}-$ sammenhang zwischen dem Recht auf Ausführung einer Funktion und einer Menge von Transaktionen her. Bereits bei der Erkundung von Angeboten erhalten Interessenten eine Übersicht über Kosten, Vorbedingungen, Transaktionen, darüber ausgedrückte Erlösmodelle sowie Folgen, die im Laufe des Lernprozesses durch ihre Anwendung auftreten. Existieren zu einer Funktion verschiedene Angebote, können Interessenten diese miteinander vergleichen, bevor sie eine Auswahl treffen. 
Grundlage für die Spezifikation von Transaktionen und Angeboten ist damit eine Angebotsverwaltung. Ebenso muss ein Zielsystem die Zuteilung von Lizenzen auf Basis dieser Angebote erlauben. Der folgende Abschnitt zeigt zusammenfassend eine Übersicht über die Erweiterung eines ursprünglichen Systems.

\section{5 Übersicht über die Gesamtarchitektur}

Grundlage für die Automatisierung von Transaktionen im E-Learning ist ein System, das eine Menge von Funktionen zur Ausführung bereitstellt. Um ihre Funktionalität vor unbefugten Zugriffen zu schützen, wird jede Funktion um Prolog und Epilog erweitert. Hinzu kommt eine Systemerweiterung, die sich mit der Verwaltung von Funktionen, Rechten und internen Konten beschäftigt. Eine Übersicht über die Zielarchitektur ist in Abb.5 illustriert.

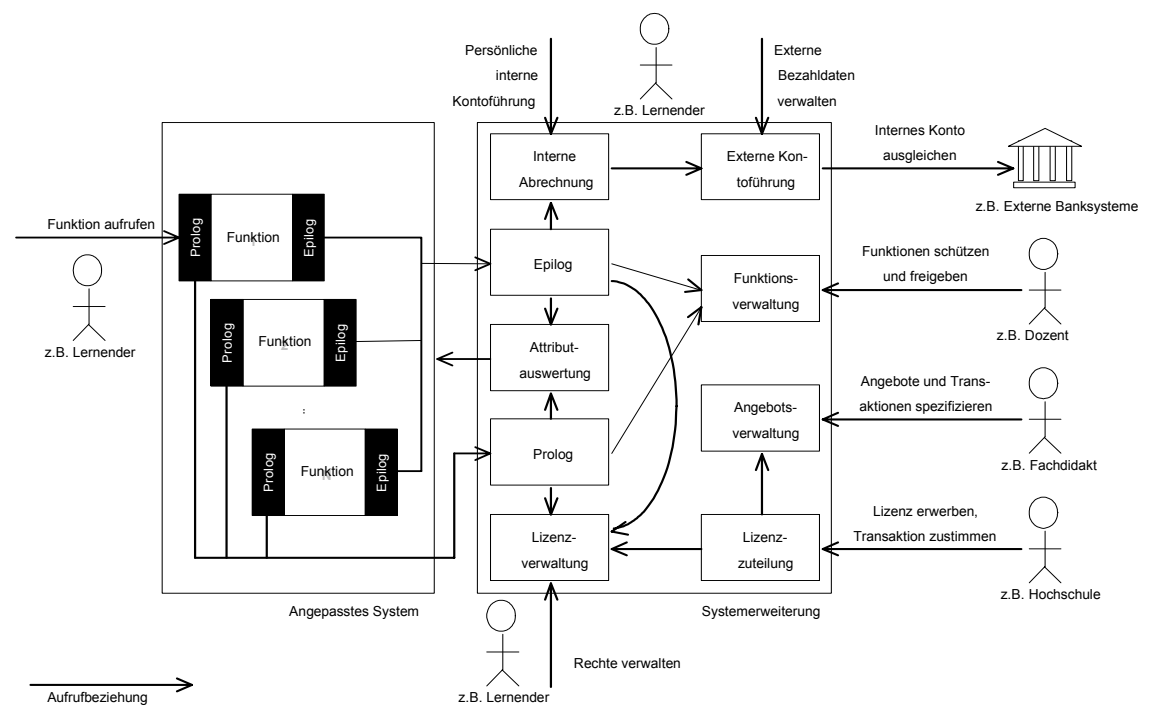

Abbildung 5: Kontrollierter Zugriff auf erweiterte Funktionen

Ruft ein Nutzer eine Funktion auf, kontaktiert der Prolog eine Systemerweiterung und prüft, ob ein Zugriff auf die Funktionalität erlaubt ist. Grundlage der Systemerweiterung ist daher eine Funktionsverwaltung, die angibt, ob eine Funktion geschützt ist, eine Rechteverwaltung, die Aussagen darüber erlaubt, unter welchen Bedingungen welcher Nutzer auf welche Funktionen zugreifen darf, sowie eine interne Abrechnung, die für die Durchführung von Transaktionen zuständig ist. Eine kurze Beschreibung von Komponenten der Systemerweiterung schließt sich nachfolgend an:

- Funktionsverwaltung: Grundlage, um Schutz und Freigabe von Funktionen auf einfache Weise vorzunehmen. Mit ihrer Hilfe überprüft der Prolog, ob ei- 
ne Funktion der Rechtekontrolle unterworfen ist und damit eine Lizenz notwendig ist, um sie auszuführen.

- Lizenzverwaltung: Zuständig für Erstellung, Änderung und Verwaltung von Lizenzen, Überprüfung der Gültigkeit sowie ihrer Beanspruchung. Sie realisiert die Rechteverwaltung, die bestimmte Nutzer, Rechte, Gültigkeiten, Vorbedingungen, Funktionen und Transaktionen miteinander verknüpft.

- Interne Abrechnung: Realisiert eine interne Kontoführung für beteiligte Nutzer oder Systeme und ist zuständig für die Durchführung finanzieller Transaktionen. Sie erlaubt zudem die Überprüfung, ob eine Durchführung von Transaktionen unter aktuellen Bedingungen möglich ist.

- Prolog und Epilog: Zentrale Komponenten, die für die Ausführung von Pro$\log$ und Epilog zuständig sind. Dies ist möglich, da Pro- und Epilog unabhängig von bestimmten Funktionen sind und ihre Ausführung immer in gleicher Weise erfolgt.

- Attributauswertung: Übernimmt im Rahmen der Ausführung von Pro- oder Epilog die Abfrage von Zuständen und Attributen des ursprünglichen Systems. Sie erlaubt eine Auswertung von Attributbedingungen und ist damit Grundlage für die Ausführung von Funktionen und Transaktionen.

- Angebotsverwaltung: Erlaubt eine Spezifikation von Angeboten, auf deren Grundlage die Erstellung von Lizenzen möglich ist. Da einzelne Transaktionen im Rahmen von Lizenzen mitverwaltet werden, dient diese Komponente ebenfalls der Spezifikation von Transaktionen. Sie ermöglicht durch ein einheitliches Format eine einfache Spezifikation, Erkundung und den Vergleich mit anderen Angeboten.

- Lizenzzuteilung: Erstellt nach Auswahl und Zustimmung zu einem Angebot aus den darin spezifizierten Attributen eine Lizenz und teilt diese dem Nutzer zu. Ist eine kostenpflichtige Zuteilung von Lizenzen vorgesehen, übernimmt die Lizenzzuteilung zusätzlich die Preisfindung.

- Externe Bezahlung: Dient dem Ausgleich eines internen Kontos und übernimmt dazu die Kommunikation mit der persönlichen Kontoführung eines Nutzers oder einer Organisation, z.B. in Form externer Banksysteme. Die Durchführung einer externen Bezahlung und damit der Ausgleich eines internen Kontos erfolgt abhängig von der innerhalb einer Transaktion spezifizierten Art der Abrechnung.

Die im Rahmen dieser Architektur vorgestellte Erweiterung eines ursprünglichen Systems erlaubt die Verwaltung von Lizenzen sowie die automatisierte Durchführung darin spezifizierter Transaktionen. Voraussetzung zum Ansprechen der Systemerweiterung und damit zum Schutz von Funktionen ist die Integration von Prolog und Epilog in ein ursprüngliches System. Der folgende Abschnitt geht darauf an einem konkreten Beispiel näher ein. 


\section{Vorgehensweise bei der Integration}

Das in dieser Arbeit vorgestellte Konzept ist weitgehend unabhängig von geschäftlichen, organisatorischen oder didaktischen Vorgaben, seine Integration daher in verschiedenartige Systeme möglich. Die Universität Oldenburg ${ }^{2}$ betreibt zur Zeit mit Stud.IP ${ }^{3}$ Version 1.1 ein serverbasiertes Open-Source LMS, das in der Programmiersprache PHP ${ }^{4}$ realisiert ist. Am Beispiel von Stud.IP soll im Folgenden kurz aufgezeigt werden, wie bei der Integration des dargestellten Konzeptes in ein konkret eingesetztes System vorzugehen ist.

- Integration von Prolog und Epilog: Die Programmiersprache PHP erlaubt nach Aufruf und vor Ausführung einer Datei zunächst die Ausführung einer in der PHP-Variablen auto prepend file angegebenen Datei. Auf diese Weise ist die Integration einer zusätzlichen Datei möglich, die ein Ansprechen und Ausführen des Prologs übernimmt. In gleicher Weise erlaubt die Belegung der PHP-Variablen auto_append_file das Zufügen eines Epilogs. Unabhängig von der Ausführung von Pro- und Epilog bleiben die aktuelle Session und der Aufruf der ursprünglichen Funktion in Form eines Requests zur weiteren Verarbeitung erhalten.

- Realisierung der Systemerweiterung: Die Realisierung der vorangehend dargestellten Komponenten kann unabhängig vom ursprünglichen System erfolgen. Ist eine Systemerweiterung z.B. in der Programmiersprache Java erstellt, können ihre Funktionen in Form von Web Services veröffentlicht und auf diese Weise über einen entsprechend ausgestatteten Pro- und Epilog mit dem ursprünglichen System gekoppelt werden. Die Attributauswertung hingegen ist die einzige Komponente mit Bezug zur internen Realisierung des ursprünglichen Systems. Sie muss dessen Attribute, Werte und Zustände abfragen können, um so zur Auswertung entsprechender Bedingungen beizutragen. Daher kann die Systemerweiterung auch in der Programmiersprache des ursprünglichen Systems realisiert und diesem zugefügt werden. Auf diese Weise resultiert zudem ein in sich geschlossenes System, das Systembrüche vermeidet.

Das Beispiel Stud.IP zeigt eine einfache Möglichkeit zur Integration des in dieser Arbeit vorgestellten Konzepts in ein bestehendes System. Jede zusätzliche Erweiterung oder Änderung des ursprünglichen Systems ist damit automatisch Teil der zugefügten Systemerweiterung und damit der gleichen Rechtekontrolle bzw. Transaktionsabwicklung unterworfen. Unter Berücksichtigung des dargestellten Konzepts beschreibt der folgende Abschnitt verwandte Arbeiten und Ansätze, die eine gewisse Beziehung und Nähe zur vorgestellten Arbeit haben.

http://www.uni-oldenburg.de/

http://www.studip.de/

http://www.php.net/ 


\section{Verwandte Arbeiten und Ansätze}

Digital Rights Management (DRM) Systeme beruhen auf geschützten digitalen Inhalten und der Beschreibung, wer unter welchen Umständen darauf zugreifen darf [ $\left.\mathrm{Bec}^{+} 03\right]$. Sobald eine zugehörige Lizenz vorhanden ist, gibt eine Einsatzumgebung (z.B. ein Player) den Zugriff auf diese Inhalte frei. Entsprechende Systeme sind auf digitale Produkte und nicht auf Dienstleistungen ausgerichtet. Ihre Integration in bestehende Abläufe des E-Learning ist daher nicht universell, sondern nur an Stellen möglich, an denen Nutzer auf Inhalte zugreifen.

Einen Ansatz zur Spezifikation von Rechten bieten Rechtebeschreibungssprachen z.B. die eXtensible rights Markup Language ${ }^{5}$ (XrML) [Cont02]. Derartige Beschreibungssprachen berücksichtigen insbesondere den Schutz digitaler Produkte [RoDy03], vernachlässigen jedoch digitale Dienstleistungen. Zudem ist XrML für das vorgestellte Szenario zu mächtig und dementsprechend unübersichtlich. Es berücksichtigt kein automatisiertes Durchführen von Transaktionen, daher wäre eine Erweiterung unumgänglich.

Zur Modellierung von Transaktionen stehen ebenfalls verschiedene Spezifikationen zur Verfügung. Die Interactive Financial Exchange ${ }^{6}$ (IFX) und die Open Financial Exchange ${ }^{7}$ (OFX) sind XML-Spezifikationen, die elektronische Rechnungslegung und Bezahlung vereinfachen. Sie haben jedoch einen anderen Anwendungsbereich, beziehen sich nämlich auf den Austausch von Bankdaten und Bankdiensten, und sind für die beabsichtigte Nutzung zu komplex.

Eine Analyse von Kosten und Nutzen im E-Learning nehmen z.B. [Seib02] oder [Kypr03] vor, mit der Erstellung von Geschäftsmodellen beschäftigen sich z.B. [Seuf01], [Bent02] oder [HoBr03], Beispiele für Geschäftsprozesse bieten z.B. [ObKi00] und [Gut $\left.{ }^{+} 01\right]$. Abgesehen von übergeordneten Ansätzen (z.B. Subventionierung) fehlt jedoch ein Instrument, das eine Umsetzung geschäftlicher Vorgaben im E-Learning erlaubt, z.B. durch ihre Integration in bestehende LMS.

Auf Grundlage eines LMS hingegen beschreiben [ReAp04] das kostenpflichtige Angebot digitaler Kursmaterialien. Sie koppeln dazu die Auslieferungsfunktion für kostenpflichtige Dateien mit einer externen Bezahlung. Sobald die Bezahlung durchgeführt wurde, wird der Verweis auf eine Datei freigegeben und diese kann durch den zahlenden Nutzer heruntergeladen werden. Eine interne Abrechnung ist nicht notwendig, da die Bezahlung direkt erfolgt. Dieser Ansatz berücksichtigt das Ansprechen externer Bezahlsysteme und den Schutz ausgewählter Materialien, ist jedoch für die Automatisierung von Transaktionen und damit für die Umsetzung komplexer ökonomischer Vorgaben nicht allgemein genug.

http://www.xrml.org/

http://www.ifxforum.org/

http://www.ofx.net/ 


\section{Zusammenfassung und Ausblick}

Auf der einen Seite hilft E-Learning dabei, bestimmte Kosten einzusparen, die z.B. durch das Versenden von Lernmaterialien beim traditionellen Fernunterricht auftreten. Auf der anderen Seite entstehen jedoch völlig neue Kosten, um z.B. eine technische Infrastruktur bereitzustellen, LMS zu betreiben, Beteiligte zu schulen, laufende Lizenzkosten zu begleichen und/oder Lerninhalte multimedial aufzubereiten. Da bisher genügend öffentliche Mittel zur Verfügung standen, erfolgte der Aufbau einer E-Learning-Infrastruktur oder von Know How in diesem Bereich bisher über Subventionierung, z.B. im Rahmen von Forschungsprojekten. Im Hinblick auf das Auslaufen dieser Förderungen sind jedoch ökonomische Überlegungen notwendig, die ein Weiterführen von E-Learning-Aktivitäten erlauben. Grundlage sind E-Learning-Geschäftsmodelle, die zunächst zu erstellen, dann aber in die E-Learning-Aktivitäten zu integrieren sind. Diese Arbeit stellt ein Konzept vor, dessen Realisierung eine Umsetzung von Erlösmodellen auf Basis bestehender ELearning-Systeme erlaubt. Es erlaubt die Kopplung finanzieller Transaktionen mit der Erbringung digitaler Dienstleistungen. Da es zudem unabhängig von der Art einer persönlichen Kontoführung ist, kann seine Erprobung auch auf Grundlage von Spielgeld oder eines Punktesystems erfolgen.

Durch Fokussierung auf eine interne Abrechnung berücksichtigt das vorgestellte Konzept bisher nur die Durchführung finanzieller Transaktionen innerhalb eines Systems. Eine Systemerweiterung kann aber genauso gut mit mehreren und verschiedenen LMS zusammenarbeiten. Sie koppelt die Systeme, indem sie die Verwaltung von Rechten und Durchführung von Transaktionen übernimmt und somit systemübergreifend als Mediator fungiert. Eine weitere Idee ist das Ansprechen externer kostenpflichtiger Funktionen. Dies ist z.B. über das Zufügen von Containerfunktionen möglich, die den Aufruf und die Ausführung externer kostenpflichtiger Funktionen übernehmen. Ein Container kann wie bereits bestehende Funktionen mit Pro- und Epilog ausgestattet werden. Im Rahmen einer PayNowAbrechnung kann dann ein Bezahlsystem angesprochen werden, das den Austausch von Zahlungsmitteln mit dem beteiligten externen System regelt.

E-Learning-Systeme und insbesondere LMS entwickeln sich mehr und mehr zur zentralen Plattform einer Hochschule. Sie dienen als Grundlage für die Integration anderer Hochschulsysteme, z.B. Prüfungsverwaltung, Lehrveranstaltungsplanung, Authentifizierung usw. Die Zusammenführung verschiedener Dienste einer oder mehrerer Hochschulen verknüpft eine Menge an Funktionalität miteinander. Der Schutz entsprechender Funktionen vor Missbrauch und ihre Integration in vorab modellierte Arbeitsabläufe ist notwendig. Das in dieser Arbeit vorgestellte Konzept bietet einen ersten Ansatz, um derartige Abläufe auf Basis eines Schutzes individueller Funktionen sowie der automatischen Durchführung finanzieller Transaktionen zu realisieren. Zudem bietet es eine einfache Möglichkeit, angedachte Erlösmodelle auf Basis bereits existierender LMS zu erproben. 


\section{Literatur}

[AdHu00] Adar, E. und Huberman, B. A.: Free Riding on Gnutella. First Monday. 5(10). Oktober 2000.

[Bec ${ }^{+}$03] Becker, E., Buhse, W., Gunnewig, D., und Rump, N.: Digital Rights Management: Technological, Economic, and Legal and Political Aspects. Lecture Notes in Computer Science. Springer Verlag. Berlin. 2003.

[Bent02] Bentlage, U.: E-Learning: Märkte, Geschäftsmodelle, Perspektiven. Bertelsmann Stiftung. Gütersloh. 2002.

[Bau 02] Baumgartner, P., Häfele, H., und Maier-Häfele, K.: E-Learning Praxishandbuch: Auswahl von Lernplattformen. Marktübersicht - Funktionen - Fachbegriffe. Österreichischer Studien Verlag. Innsbruck. 2002.

[Bal ${ }^{+}$02] Balli, C., Krekel, E. M., und Sauter, E.: Qualitätsentwicklung in der Weiterbildung: Zum Stand der Anwendung von Qualitätssicherungs- und Qualitätsmanagementsystemen bei Weiterbildungsanbietern. Schriftenreihe des Bundesinstituts für Berufsbildung, Heft 62. 2002.

[Bole02] Boles, D.: Integration von Konzepten und Technologien des Electronic Commerce in digitale Bibliotheken. 2002. Dissertation, Universität Oldenburg.

[BaPa99] Baumgartner, P. und Payr, S.: Lernen mit Software. Reihe Digitales Lernen. Österreichischer Studien Verlag. Innsbruck. 2. 1999.

[BrSt02] Bruhn, M. und Stauss, B.: Electronic Services. Gabler Verlag. Wiesbaden. 2002.

[BoSc03] Boles, D. und Schmees, M.: Kostenpflichtige Web-Services. In: Uhr, W., Esswein, W., und Schoop, E. (Hrsg.),Wirtschaftsinformatik 2003: Märkte - Medien - Mobilität. Band I. S. 385-403. Physica Verlag. Heidelberg. 2003.

[Cont02] Contentguard: XrML 2.0 Technical Overview. 2002. Version 1.0.

[Gol $\left.{ }^{+} 01\right]$ Golle, P., Leyton-Brown, K., und Mirnov, I.: Incentives for Sharing in Peer-toPeer Networks. In: Proceedings of the 2001 ACM Conference on eCommerce. 2001.

[Gut ${ }^{+} 01$ ] Guth, S., Neumann, G., und Simon, B.: Universal - design spaces of learning media. In: Sprague, R. H. (Hrsg.), Proceedings of the 34th Annual Hawaii International Conference on System Sciences.Volume 7. IEEE Computer Society. Maui. 2001.

[Habe03] Haber, C.: COMMA - eine Entwicklungsmethode für interaktive kooperative Anwendungen. dissertation.de. Berlin. 2003. Dissertation, Universität Oldenburg.

[HoBr03] Hoppe, G. und Breitner, M. H.: Business Models for E-Learning. (287). Oktober 2003.

[HaOt02] Hasebrook, J. und Otte, M.: E-Learning im Zeitalter des E-Commerce: die dritte Welle. Hans Huber Verlag. Bern. 2002.

[Jung03] Jung, H.W.: Praxisorientiertes Geschäftsmodell. In: Ehlers, U.-D., Gerteis, W., Holmer, T., u. a. (Hrsg.), E-Learning-Services: im Spannungsfeld von Pädagogik, Ökonomie und Technologie. S. 465-477. Bertelsmann Verlag. Bielefeld. 2003. 
[Kypr03] Kypreos, T.: Building a Business Case for E-Learning. In: eLearn-Magazine. 2003.

[Leh ${ }^{+}$03] Lehner, F., Schäfer, K. J., und Proksch, M.: Was kostet e-Learning. In: Bode, A., Desel, J., Rathmayer, S., u. a. (Hrsg.), DeLFI 2003: Tagungsband der 1. e-Learning Fachtagung Informatik. S. 240-249. Köllen Druck \& Verlag GmbH. Bonn. 2003.

[Luxe01] Luxem, R.: Digital Commerce: Electronic Commerce mit digitalen Produkten. Josef Eul Verlag. Lohmar. 2. 2001. Dissertation, Universität Münster.

[Mass02] Massy, J.: Quality and eLearning in Europe: Summary report 2002. Umfrage von BIZmedia. Juni 2002.

[Mina02] Minass, E.: Dimensionen des E-Learning: Neue Blickwinkel und Hintergründe für das Lernen mit dem Computer. SmartBooks Publishing. Kilchberg. 2002.

[Moon97] Moonen, J.: The Efficiency of Telelearning. JALN: Journal of Asynchronous Learning Networks. 1(2). August 1997.

[ObKi00] Oblinger, D. und Kidwell, J.: Distance learning - are we being realistic? Educause Review. 35. Mai/Juni 2000.

[Oste04] Osterwalder, A.: The Business Model Ontology: A Proposition in a Design Science Approach. 2004. Dissertation, Universität Lausanne.

[ReAp04] Reil, D. und Appelrath, H.-J.: Kostenpflichtiger Content in Lernportalen. In: Engels, G. (Hrsg.), DeLFI 2004: Tagungsband der 2. e-Learning Fachtagung Informatik. Paderborn. 2004.

[RoDy03] Rosenblatt, B. und Dykstra, G.: Content Management with Digital Rights Management: Imperatives and Opportunities for Digital Content Lifecycles. 2003.

[Rumb01] Rumble, G.: The Costs and Costing of Networked Learning. JALN: Journal of Asynchronous Learning Networks. 5(2). September 2001.

[Seuf01] Seufert, S.: E-learning business models: Strategies, success factors and best practice examples. In: DeFillippi, R. und Wankel, C. (Hrsg.), Rethinking Management Education for the 21st Century. Information Age Publishing. Greenwich. 2001.

[Seib02] Seibt, D.: Kosten und Nutzen des E-Learning bestimmen. In: Hohenstein, A., Wilbers, K. (Hrsg.): Handbuch E-Learning: Expertenwissen aus Wissenschaft und Praxis. S. 130-162. Fachverlag Deutscher Wirtschaftsdienst. Köln. 2002.

[ScHe02] Scheffer, U. und Hesse, F. W.: E-Learning: Die Revolution des Lernens gewinnbringend einsetzen. Klett-Cotta Verlag. Stuttgart. 2002.

[Stei01] Steiner, G.: Lernen und Wissenserwerb. In: Krapp und Weidenmann (Hrsg.), Pädagogische Psychologie: ein Lehrbuch. S. 160-163. Beltz Verlag. Weinheim. 4. 2001.

[Timm98] Timmers, P.: Business Models for Electronic Markets. Electronic Markets: Electronic Commerce in Europe. 8(2):3-8. 1998.

[Weng00] Wengelowski, P.: Entwicklung organisationalen Lernens: ein Lenkungsmodell. Dissertation. Universität Oldenburg. Deutscher Universitätsverlag. Wiesbaden. 2000. 